\title{
AUTOSOMAL-RECESSIVE NEPHROGENIC DIABETES INSIPIDUS ASSOCIATED WITH INTRACRANIAL CALCIFICATIONS, MENTAL RETARDATION
}

The department of faculty pediatrics Saint-Petersburg State Pediatric Medical University, Russia

РЕФЕРАТ

В статье представлены клинические наблюдения двоих пациентов с редкой ассоциацией аутосомно-рецессивного нефрогенного несахарного диабета с кальцификатами головного мозга и снижением интеллекта.

Ключевые слова: аутосомно-рецессивный нефрогенный несахарный диабет, кальцификаты головного мозга, снижение интеллекта.

\section{ABSTRACT}

The article presents case histories of two patients with a rare association of autosomal-recessive nephrogenic diabetes insipidus with intracranial calcifications and mental retardation.

Key words: autosomal-recessive nephrogenic diabetes insipidus, intracranial calcifications, mental retardation.

Врожденный нефрогенный несахарный диабет (ВННД) или почечный несахарный диабет - наследственное заболевание, ведущими симптомами которого являются полиурия, полидипсия, гипостенурия [1,2]. Терапия тиазидными диуретиками даёт парадоксальный эффект снижения полиурии и полидипсии у пациентов с врожденным нефрогенным несахарным диабетом (ВННД). Пациентам с ВННД применяются: изолированная терапия гипотиазидом, комбинированная терапия гипотиазидом и индометацином с препаратами калия, комбинированная терапия гипотиазидом и калийсберегающим диуретиком амилоридом.

Актуальность проблемы аутосомнорецессивного нефрогенного несахарного диабета в ассоциации с интракраниальными кальцификатами у детей обусловлена развитием синдрома полиурии, полидипсии, гипостенурии и осложнений (мегацистис, гидронефроз без анатомической обструкции, ХБП) в сочетании с кальцификатами

Савенкова Н.Д. 194100, Санкт-Петербург, ул. Литовская, д. 2. ФГБОу ВО Санкт-Петербургский государственный педиатрический медицинский университет Минздрава России, кафедра факультетской педиатрии. Тел.: (812) 416-52-86, E-mail: savenkova.n.spb@mail.ru головного мозга, сопровождающимися снижением интеллекта, неврологическими нарушениями.

Аутосомно-рецессивный нефрогенный несахарный диабет (ННД) с интракраниальными кальцификатами и снижением интеллекта выделен в самостоятельную нозологическую форму и обозначен в систематике по ОМІМ - 221995 [3, 4].

В отечественной и зарубежной литературе используется следующая терминология: ННД с интракраниальными кальцификатами, задержкой психического развития (nephrogenic diabetes insipidus with intracranial calcification, mental retardation), ННД с интрацеребральными кальцификатами (nephrogenic diabetes insipidus with intracerebral calcification), ННД с церебральными кальцификатами и снижением интеллекта (nephrogenic diabetes insipidus with cerebral calcification and mental retardation) [3-17].

С момента описания аутосомно-рецессивного ННД с кальцификатами головного мозга в 1983 году как редкого синдрома [5], в зарубежной литературе представлено всего 20 случаев этой ассоциации у детей в возрасте от 50 дней до 18 лет [5-17] (табл. 1). 
В связи с отсутствием описания приводим клиническое наблюдение двоих пациентов с редким синдромом - аутосомно-рецессивным нефрогенным несахарным диабетом в ассоциации с билатеральными интракраниальными кальцификатами, снижением интеллекта.

Клиническое наблюдение 1.

Пациент $\Phi$. от IV беременности (I-III беременности закончились спонтанными абортами), протекавшей на фоне постоянной угрозы прерывания. C первых недель жизни у ребёнка отмечались характерные симптомы ВННД - полидипсия, полиурия, длительные фебрильные лихорадки, а также аффективно-респираторные пароксизмы, анемия III степени (по поводу чего получал гемотрансфузии). В 6 мес. перенес вирусный гепатит В, выявлены признаки гипоксически-ишемической энцефалопатии, отставание в физическом и психомоторном развитии, неоднократно госпитализировался с подозрением на гнойно-септические заболевания. С 8,5 месяцев мальчик наблюдался нефрологом с изолированным диагнозом проксимальный почечный канальцевый ацидоз II типа. В 5 лет у мальчика при обследовании выявлены полиурия/полидипсия (до 5 л/сут), гипостенурия (1000), диагностирован ВННД, назначена терапия гипотиазидом (0,008 г 3 p/сут), в результате которой диурез снизился до 4 л/сут. До терапии пациенту была проведена проба с синтетическим аналогом вазопрессина (DDAVP) которая не привела к снижению полиурии и полидипсии, повышению относительной плотности и осмоляльности мочи. У мальчика в 6 лет выявлены осложнения ВННДмегацистис. Начата комбинированная терапия гипотиазидом и индометацином с препаратами калия, в результате терапии у мальчика диурез снизился до 3,5-4,5 л/сут. С 10 лет пациент получал комбинированную терапию гипотиазидом (3 мг/ кг/сут) и калийсберегающим диуретиком амилоридом (0,3 мг/кг/сут) с положительным эффектом. В 13 лет у пациента полиурия/полидипсия достигали 8-9 л/сут, в 15 лет - 10-12 л/сут, выявлена гиперкальциурия (472 мг/сут). Родители отказались от дальнейшей фармакотерапии. В 16 лет у пациента полиурия и полидипсия составляли 16-17 л/сут. Выявлено отставание в умственном развитии, нарушение осанки, сколиоз. По результатам рентгено-урологического обследования выявлена гидронефротическая трансформация без признаков анатомической обструкции. По результатам КТ обнаружены у пациента симметричные перивентрикулярные в лобных и затылочных долях

Таблица1

Обзор описанных в литературе случаев ННД с интракраниальными кальцификатами [5-17]

\begin{tabular}{|c|c|c|c|}
\hline Авторы & $\begin{array}{l}\text { Возраст ребёнка } \\
\text { при обследовании }\end{array}$ & $\begin{array}{l}\text { Снижение } \\
\text { интеллекта }\end{array}$ & Область интракраниальной кальцификации \\
\hline Miura et al. 1983 [5] & 5 лет, 3 года & не известно & двусторонняя в базальных ганглиях и лобных долях \\
\hline Kanzaki et al. 1985 [6] & 2 года 11 мес & + & $\begin{array}{l}\text { двусторонняя симметричная в белом веществе от лобной до } \\
\text { затылочной доли }\end{array}$ \\
\hline Ohno M. et al. 1988 [7] & 2 года & - & двусторонняя в базальных ганглиях, лобныхи затылочных долях \\
\hline \multirow[t]{2}{*}{ Freycon M.T. et al. 1988 [8] } & 16 лет & + & двусторонняя в базальных ганглиях и лобных долях \\
\hline & 18 лет & + & двусторонняя в базальных ганглиях и лобных долях \\
\hline \multirow[t]{4}{*}{$\begin{array}{l}\text { Schofer et al. } \\
1990 \text { [9] }\end{array}$} & 10 лет & + & $\begin{array}{l}\text { диссеминированная в белом веществе и } \\
\text { областях коры }\end{array}$ \\
\hline & 14 лет & + & массивная интракраниальная \\
\hline & 6 лет & + & симметрично в белом веществе лобной доли \\
\hline & 7 мec. & + & $\begin{array}{l}\text { массивные двусторонние в лобной, теменной и затылочной } \\
\text { долях }\end{array}$ \\
\hline \multirow[t]{2}{*}{ Tohyama et al. 1993 [10] } & 2 года 10 мес. & + & в базальных ганглиях, подкорковом белом веществе \\
\hline & 8 мec. & + & в базальных ганглиях, подкорковом белом веществе \\
\hline Nozue et al. 1993 [11] & 6 лет & + & $\begin{array}{l}\text { в базальных ганглиях, лобной, теменной, височной и затылоч- } \\
\text { ной областях }\end{array}$ \\
\hline \multirow[t]{2}{*}{ Bagga et al. 1996 [12] } & 5 мec. & + & симметричные в базальных ганглиях \\
\hline & 2 года & + & в лобных долях и базальных ганглиях \\
\hline Zaki et al. 1997 [13] & 50 дней & - & $\begin{array}{l}\text { двусторонние в базальных ганглиях и на стыке белого и серого } \\
\text { вещества в теменной доле }\end{array}$ \\
\hline Ray M. et al. 2002 [14] & 9 мес. & - & двусторонняя в базальных ганглиях \\
\hline Bajpai A.et al. $2005[15]$ & 1 год & + & двусторонние в лобных долях, базальных ганглиях и таламусе \\
\hline Bindu P.S.et al. 2007 [16] & 4 года & + & $\begin{array}{l}\text { двусторонние в белом веществе лобных и теменных областей, } \\
\text { теменных и затылочных долях }\end{array}$ \\
\hline Dimple J.et al. 2013 [17] & 12 лет & + & массивная интракраниальная \\
\hline
\end{tabular}




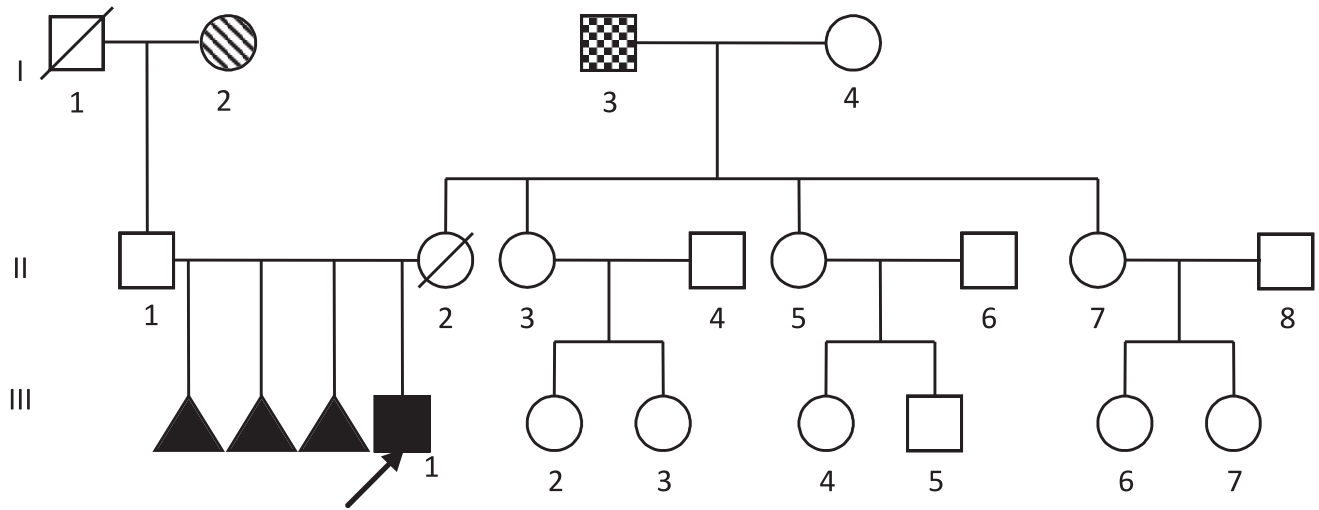
спонтанный аборт
нефрогенный несахарный диабет
$\$$ артериальная гипертензия
бронхиальная астма

Рис.1. Фрагмент родословной семьи Ф.

множественные кальцификаты до 1 см в диаметре и мелкие без четких границ - в области подкорковых ядер. Пациенту в 17 лет назначена терапия гипотиазидом (3 мг/кг/сут) с препаратами калия и индометацином (2 мг/кг/сут), в результате чего диурез снизился до 8-10 л/сут, в 23 года диурез составил 17 л/сут. Органическое поражение головного мозга прогрессировало: снижение интеллектуального уровня, грубые нарушения письма, умеренные нарушения чтения, дизартрия. Фрагмент родословной семьи Ф. представлен на рис. 1.

Диагноз клинический: аутосомно-рецессивный нефрогенный несахарный диабет, ассоциированный с множественными симметричными кальцификатами перивентрикулярными в лобных и затылочных долях головного мозга и снижением интеллекта. Ожирение II степени смешанной этиологии.

Клиническое наблюдение 2.

Пациентка С. от I беременности, протекавшей без особенностей, роды физиологические в срок. В неонатальном возрасте у девочки отмечались немотивированные подъемы температуры, рвота, признаки вододефицитной дегидратации с гипернатриемией, судороги, отставание в физическом и психомоторном развитии, в 3 месяца - эпизод клинической смерти. В возрасте 1 года девочке установлен диагноз врожденный нефрогенный несахарный диабет и выявлены билатеральные кальцификаты в базальных ганглиях головного мозга, пролапс митрального и трикуспидального клапанов. При обследовании у девочки в 14 лет клинические признаки ВННД - полиурия, полидипсия (15-18 л/ сут), гипостенурия (1000), снижение интеллекта, алалия, ожирение III степени смешанного гене- за. Проба с DDAVP не дала снижения количества выпиваемой и выделяемой жидкости, повышения относительной плотности и осмоляльности мочи. Диагностированы осложнения: двухсторонний мегауретер, мегацистис без признаков анатомической обструкции. Данные исследований: осмоляльность плазмы крови в пределах нормы (282 мосмоль/ кг $\mathrm{H}_{2} \mathrm{O}$ ), низкая осмоляльность мочи 45 (мОсмоль/ кг $\mathrm{H}_{2} \mathrm{O}$ ), гипофосфатемия 1,06 ммоль/л, гипокальцемия (2,15 ммоль/л), гиперкальциурия (711 мг/ сутки), по пробе Реберга скорость клубочковой фильтрации 154 мл/мин, канальцевая реабсорбция 95,5\% (при диурезе 10 л/сут), гипохлоремический метаболический алкалоз (Cl 98 ммоль/л). В 16 лет у девочки выявлены кальцификаты лимфатических узлов средостения и перикарда (диаметр 15 мм). С момента постановки диагноза ВННД в возрасте 1 года до 13 лет девочка получала изолированную диуретическую терапию гипотиазидом (3 мг/кг в сутки) с препаратами калия ежедневно, с 14 лет комбинированную терапию гипотиазидом (3 мг/кг в сутки) и амилоридом ( 0,3 мг/кг в сутки) ежедневно, на фоне которой наблюдалось снижение полиурии/полидипсии (с 10 л/сут до 1 л/сут). Фрагмент родословной семьи С. представлен на рис. 2.

Диагноз клинический: аутосомно-рецессивный нефрогенный несахарный диабет, ассоциированный с множественными симметричными кальцификатами в базальных ганглиях головного мозга и снижением интеллекта. Ожирение III степени смешанной этиологии.

\section{ОБСУЖДЕНИЕ}

Клинические наблюдения демонстрируют у 


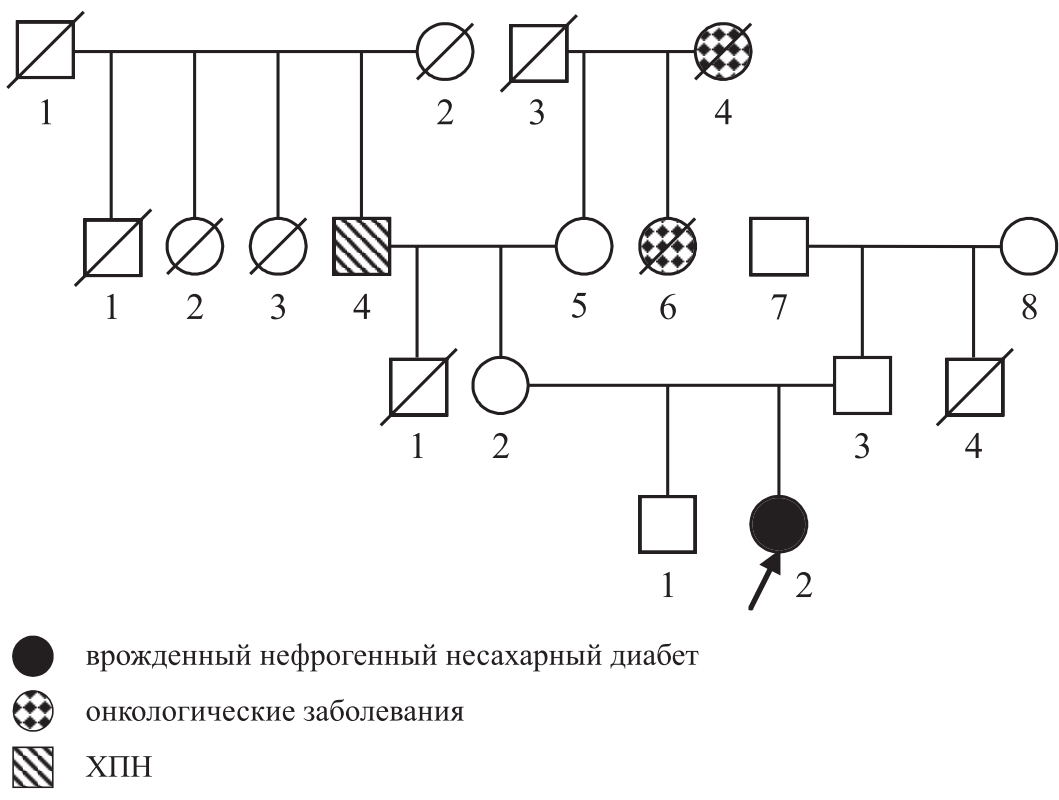

Рис. 2. Фрагмент родословной семьи С.

двоих пациентов редкую ассоциацию аутосомнорецессивного ННД с двусторонними симметричными кальцификатами головного мозга, снижением интеллекта, ожирением. У одной девочки кроме симметричных кальцификатов головного мозга выявлены кальцификаты лимфатических узлов средостения и перикарда, метаболический алкалоз (наблюдение 2).

Особенностью катамнестического наблюдения пациента Ф. с ННД является позднее обнаружение кальцификатов головного мозга в 16 лет. У пациентки С. с ВННД кроме кальцификатов головного мозга, диагностированных в возрасте 1 года, отмечено возникновение кальцификатов внутригрудных лимфатических узлов и перикарда, метаболического гипохлоремического алкалоза в 16 лет. По данным зарубежных авторов, интракраниальные кальцификаты, выявляемые у пациентов с ННД, в большинстве случаев двусторонние и симметричные, что отмечено у описываемых нами пациентов [5-17]. Одни авторы предполагают, что появление интракраниальных кальцификатов может быть объяснено повторными эпизодами вододефицитных дегидратаций в раннем возрасте, приводящих к некрозу эндотелиальных клеток с последующей кальцификацией вокруг очага некроза $[5,9]$, частыми эпизодами гипернатриемии, которые могут приводить к поражению головного мозга - петехиальным геморрагиям в кортикальном слое и субкотрикальном белом веществе, тромбозам капилляров и сосудов [18]. A. Bagga et al. (1996) подчеркивает, что интракраниальные кальцификаты у больных ННД прогрессируют, несмотря на адекватную терапию, и приводят к снижению интеллекта [12]. Выявление двусторонних кальцификатов головного мозга у пациентки С. с раннего возраста обусловлено, по-видимому, отложением кальция во внутриутробном периоде или эпизодами гипернатриемической дегидратации. Длительная терапия гипотиазидом дает побочные эффекты - гиперкальциемию, гипохлоремический метаболический алкалоз и отложение кальция в стенках сосудов и мягких тканях [19]. Длительное применение гипотиазида у девочки C. с ННД и интракраниальными кальцификатами могло привести к развитию кальцификатов внутригрудных лимфоузлов и перикарда, метаболическому алкалозу, а также к развитию поздних интракраниальных кальцификатов у пациента $\Phi$. Другие авторы в опубликованных наблюдениях описывают случаи ННД с интракраниальными кальцификатами у детей первых 9 месяцев, ещё не получавших терапию диуретиками $[10,12,13$, 14]. До настоящего времени механизм развития интракраниальных кальцификатов остаётся неизвестным.

Для исключения интракраниальных кальцификатов у больных с нефрогенным несахарным диабетом с аутосомно-рецессивным типом необходимо проводить компьютерную томографию головного мозга. При лечении пациентов с ВННД тиазидными диуретиками целесообразно проводить контроль КОС, калия, кальция в крови и определение суточной кальциурии. С целью предупреждения развития вододефицитного обезвоживания с гипернатриемией рекомендовано не ограничивать пациентов с аутосомно-рецессивным ННД с кальцификатами в приёме жидкости, обеспечи- 
вать суточный объем жидкости по диурезу и гипосолевую диету, при развитии вододефицитной дегидратации своевременно проводить оральную и инфузионную регидратационную терапию.

\section{ЗАКЛЮЧЕНИЕ}

Аутосомно-рецессивный нефрогенный несахарный диабет в ассоциации с интракраниальными кальцификатами и задержкой психического развития (ОМІМ - 221995) у детей клинически проявляется полиурией, полидипсией, гипостенурией с рождения, с развитием двусторонних симметричных кальцификатов головного мозга в грудном, дошкольном и школьном возрасте, снижением интеллекта.

\section{БИБЛИОГРАФИЧЕСКИЙ СПИСОК}

1. Knoers N, Levtchenko EN. Nephrogenic diabetes insipidus. In: ED Avner, WE Harmon, P Niaudet, N Yoshikawa. Pediatric nephrology: 6-th edition. Berlin-Heidelberg: Springer-Verlag, Vol. 1, Sections 1-6, 2009; 1005-1018. ISBN 978-3-540-76341-3

2. Linshaw MA. Back to Basics: Congenital nephrogenic diabetes insipidus. Pediatrics in Review 2007;28:372-380. doi: 10.1542/pir.28-10-372

3. OMIM: An online catalog of human genes and genetic disorders [Electronic resource]. Electronic data. Baltimore: Johns Hopkins Univ., 2015. Mode of access: http://www.moim.org/, free. Title of screen

4. Левиашвили ЖГ, Савенкова НД. Справочник по наследственным синдромам с патологией почек удетей. Под. ред. Н.Д. Савенковой. «Левша. Санкт-Петербург», СПб, 2015; 104c [Leviashvili ZhG, Savenkova ND. Spravochnik po nasledstvennym sindromam s patologiej pochek u detej. Pod. red. N.D. Savenkovoy. "Levsha. Sankt-Peterburg», SPb, 2015; 104s]

5. Miura J, Tachi N, Okabe M, Sogawa H. Two cases of nephrogenic diabetes insipidus associated with intracranial calcification (in Japanese). Acta Paediatr Jpn. 1983; 87:934-938.

6. Kanzaki S, Omura T, Miyake M et al. Intracranial calcification in nephrogenic diabetes insipidus. JAMA 1985;254(23):33493350. doi: 10.1001/jama.254.23.3349

7. Ohno M, Nakao K, Nozue T et al. A case of nephrogenic diabetes insipidus with intracerebral calcification. No To Hattatsu 1988;20(1):23-27. doi: 10.11251/ojjscn1969.20.23

8. Freycon MT, Lavocat MP, Freycon F. Familial nephrogenic diabetes insipidus with chronic hypernatremia and cerebral calcifications. Pediatrie 1988;43(5):409-413

9. Schofer O, Beetz R, Kruse K et al. Nephrogenic diabetes insipidus and intracerebral calcification. Arch Dis Child 1990;65(8):885-887. doi: 10.1136/adc.65.8.885

10. Tohyama J, Inagaki M, Koeda Tet al. Intracranial calcification in siblings with nephrogenic diabetes insipidus: CT and MRI Neuroradiology 1993;35(7):553-555. doi: 10.1007/BF00588723

11. Nozue T, Uemasu F, Endoh $\mathrm{H}$ et al. Intracranial calcifications associated with nephrogenic diabetes insipidus. Pediatr Nephrol 1993;7(1):74-76. doi: 10.1007/BF00861577

12. Bagga A, Kumar A, Bajaj G et al. Intracranial calcification in nephrogenic diabetes insipidus. Clin Pediatr (Phila) 1996;35(1):34-36. doi: 10.1177/000992289603500107

13. Zaki M, Ismail EA, Nadi HM, Nour el Din A. Recurrent dehydration in a young girl. Postgrad Med J 1997;73(860):367-369. doi: $10.1136 /$ pgmj.73.860.367

14. Ray M, Dixit A, Singhi P. Nephrogenic diabetes insipidus with intracranial calcifications. Indian Pediatr 2002;39(2):197-202

15. Bajpai A, Kabra M, Thapliyal R et al. Nephrogenic diabetes insipidus presenting with developmental delay and intracranial calcification. Indian J Pediatr 2005;72(6):527-528. doi: 10.1007/ BF02724433

16. Bindu PS, Kovoor JM. Nephrogenic diabetes insipidus: a rare cause of intracranial calcification in children. J Child Neurol 2007;22(11):1305-1307. doi: 10.1177/0883073807307087

17. Dimple J, Alka J, Mona G, Atul D. Nephrogenic diabetes insipidus with intracranial calcification in a child with thalassemia minor. Arab J Nephrol Transplant 2013;6(3):185-187

18. Conley SB. Hypernatremia. Pediatr Clin North Am 1990; 37:365-372

19. Михайлов ИБ, Маркова ИВ. Медикаментозные осложнения и пути их устранения. Диля, СПб, 2004; $123-$ 126,164-167 [Mihajlov IB, Markova IV. Medikamentoznye oslozhneniya i puti ih ustraneniya. DILYA, SPb, 2004; 123-126,164-167]

\section{Сведения об авторах:}

Ассистент Семенова Оксана Александровна, к.м.н.

194100, Санкт-Петербург, ул. Литовская, д. 2. ФГБОУ ВО СанктПетербургский государственный педиатрический медицинский университет Минздрава России, кафедра факультетской педиатрии. Тел.: (812) 416-52-86, E-mail: semdoc@bk.ru Assistant professor Oksana A. Semenova MD, PhD Affiliations: 194100, Russia, St-Petersburg, Litovskaya st., 2. Saint-Petersburg State Pediatric Medical University Ministry of Health of the Russian Federation. Department of faculty pediatrics. Phone (812) 416-52-86; E-mail: semdoc@bk.ru

Аассистент Степанова Арина Александровна

194100, Санкт-Петербург, ул. Литовская, д. 2. ФГБОУ ВО Санкт-Петербургский государственный педиатрический медицинский университет Минздрава России, кафедра факультетской педиатрии. Тел.: (812) 416-52-86, E-mail: ariwka@list.ru Assistant professor Arina A. Stepanova MD

Affiliations: 194100, Russia, St-Petersburg, Litovskaya st., 2. Saint-Petersburg State Pediatric Medical University Ministry of Health of the Russian Federation. Department of faculty pediatrics. Phone (812) 416-52-86; E-mail: ariwka@list.ru

Проф. Савенкова Надежда Дмитриевна, д.м.н.

194100, Санкт-Петербург, ул. Литовская, д. 2. ФГБОУ ВО Санкт-Петербургский государственный педиатрический медицинский университет Минздрава России, заведующая кафедрой факультетской педиатрии. Тел.: (812) 416-52-86, E-mail: savenkova.n.spb@mail.ru

Prof. Nadezda D. Savenkova MD, PhD, DMedSci

Affiliations: 194100, Russia, St-Petersburg, Litovskaya st., 2. SaintPetersburg State Pediatric Medical University Ministry of Health of the Russian Federation. Head of department of faculty pediatrics. Phone (812) 416-52-86; E-mail: savenkova.n.spb@mail.ru

Наталья Юрьевна Наточина, канд.мед.наук, доцент кафедры факультетской педиатрии ФГБОУ ВО СПбГПМУ Минздрава России, адрес: 194100, Санкт-Петербург, Литовская, 2. телефон: 416-52-86. E-mail:smallnataly@mail.com.

Natalya V. Natochina, MD PhD, Department of Faculty Pediatric of Saint-Petersburg State Pediatric Medical University Ministry of Health of the Russian Federation. 194100, Russia, StPetersburg, Litovskaya st., 2. Phone (812) 416-52-86; E-mail: E-mail:smallnataly@mail.com.

Авторы заявляют об отсутствии конфликта интересов.

Поступила в редакцию: 28.10.2016 г. Принята в печать: 31.03 .2017 г. 